\title{
The two generator restricted
}

\section{Burnside group of exponent five}

\section{George Havas, G.E. Wall, and J.W. Wamsley}

The two generator restricted Burnside group of exponent five is shown to have order $5^{34}$ and class 12 by two independent methods. A consistent commutator power presentation for the group is given.

\section{Introduction}

In 1902 Burnside [4] wrote "A still undecided point in the theory of discontinuous groups is whether the order of a group may be not finite while the order of every operation it contains is finite". This leads to the following problem, now called the Burnside problem: "If a group is finitely generated and of finite exponent, is it finite?" This is a very difficult question so a weaker form known as the restricted Burnside problem has also been investigated. The restricted Burnside problem is "Given integers $n$ and $r$ is there a largest finite group $\bar{B}(n, r)$ with $r$ generators and exponent $n$ ?" Of course an affirmative answer to the Burnside problem is automatically an affirmative answer to the restricted Burnside problem.

Let $F_{r}$ be the free group with $r$ generators and let $N$ be the normal subgroup of $F_{p}$ generated by all elements $x^{n}, x$ in $F_{p}$, then $B(n, r)=F_{r} / N$ is clearly an $r$ generator group of exponent $n$. Further every $r$ generator group of exponent $n$ is a homomorphic image of $B(n, r)$ so the unrestricted problem may be stated "Are the groups $B(n, r)$

Received 25 March 1974. The third author was partly supported by the IBM Systems Development Institute, Canberra. 
finite?" It is known that the groups $B(2, r), B(3, r), B(4, r)$ and $B(6, r)$ are finite. On the other hand Novikov and Adyan $[11,12,13]$ have proved that, for all odd $n$ not less than 4381 and all $r$ greater than $1, B(n, p)$ is infinite. More recently Adyan [1] has announced that the bound on $n$ has been reduced to 665 .

For the prime exponent case of the restricted Burnside problem the relationship between groups and Lie algebras can be utilized because the (p-1)th Engel relation holds. Kostrikin [8] proved that a Lie algebra of characteristic $p$ satisfying the $m$ th Engel condition, for $m$ less than $p$, is locally nilpotent. Consequently, the restricted Burnside problem is answered affirmatively for prime exponent.

Even in cases where the Burnside group $B(n, r)$ is known to be finite or the restricted Burnside group $\bar{B}(n, r)$ is known to exist, sometimes little more is known. For example, the orders of $B(2, r), B(3, r)$ and $B(6, r)$ are known for all $r$ but until recently the order of $B(4, r)$ has been unknown for $r$ greater than 2 while the order of $\bar{B}(p, r)$ has been unknown for all prime $p$ greater than 4 and $r$ greater than 1 .

Utilizing the relationship between groups and Lie algebras, Kostrikin [6] showed that $5^{31} \leq|\bar{B}(5,2)| \leq 5^{34}$ and that $\bar{B}(5,2)$ has class at most 12 by showing that the two generator free Lie algebra of characteristic 5 satisfying the 4 th Engel condition, $E(5,2)$, satisfies $5^{31} \leq|E(5,2)| \leq 5^{34}$, that the largest class 10 homomorphic image of $E(5,2)$ has order $5^{31}$, and that $E(5,2)$ has class at most 12 . Later, by purely combinatorial means, Kostrikin [7] determined other bounds on Engel algebras. For example he showed that the class of $E(7,2)$ was at least 18 and that the largest class 14 homomorphic image of $E(7,2)$ has order $7^{1075}$. This provides a lower bound for $|\bar{B}(7,2)|$.

Recently Bayes, Kautsky and Wamsley [3] have determined that the order of $B(4,3)$ is $2^{69}$ and that the group has class 7 by computing a consistent commutator power presentation for that group. Work is currently in progress on computing a consistent commutator power presentation for $B(4,4)$ (Alford, Havas and Newman [2]).

In this paper we describe the application of Lie algebraic methods and nilpotent group calculation methods to an investigation of $\bar{B}(5,2)$. We briefly describe the methods and present a complete multiplication table 
for $\bar{B}(5,2)$ in terms of a consistent commutator power presentation.

\section{Lie algebraic methods}

The key to the application of Lie algebras to investigation of restricted Burnside groups is given by the results of Sanov [14] and Kostrikin [7]. Sanov showed that the lie ring of $\bar{B}(p, r)$ corresponds exactly to $E(p, r)$ to class $2 p-2$. Kostrikin extended the result by showing that for 2 generators the correspondence extends to class $2 p$.

Krause and Weston [9] and Havas [5] determined that $E(5,2)$ has order exactly $5^{34}$ and class 12 , in different ways. Krause and Weston found a matrix representation for $E(5,2)$ based on Kostrikin's original calculations while Havas used a general purpose computer program for calculating Lie algebras to calculate $E(5,2) a b$ initio. These calculations alone do not throw significantly more light on $\bar{B}(5,2)$ by virtue of the problem of the correspondence between the Engel algebra and the associated Lie ring of $\bar{B}(5,2)$ at classes 11 and 1.2 .

The final step was provided by Wall in an as yet unpublished refinement to the work described in [16]. Investigating the correspondence between the Lie ring, $L(p, r)$, of $\bar{B}(p, r)$ and $E(p, r)$ he showed that, in general, additional relations hold between commutators in $L(p, r)$ beyond those that hold in $E(p, r)$. Specifically for $p$ equal to 5,7 and 11 and $r$ greater than 2 he showed that at class $2 p-1$ some of these relations are nontrivial modulo $E(p, r)$.

What Wall did in fact was determine certain additional relations which hold in Lie rings associated with prime exponent groups. Utilizing Hughes' groups constructions (see Wall [15]) he proved their independence from the Engel relations in the specific cases mentioned.

Wall's relations start at weight $2 p-1$ and exist at that and higher weights. For weights up to $3 p-3$ Wall has shown that no further relations are necessary provided that the distinct Kostrikin symbols of weight $p-1$ less than the given weight are independent in the free Lie algebra.

The distinct Kostrikin symbols for weights 7 and 8 are independent in the two generator free Lie algebra over $G F(5)$. This is proved by Kostrikin [7] and can also easily be seen from Havas cumputations. 
It follows that the addition of Wall's relations to $E(5,2)$ will give $L(5,2)$. The relations are as follows.

Wall defined a particular associative polynomial $f(x, y)$, comprising monomials of weight 8 over $\mathrm{GF}(5)$, containing 70 terms. If $f(x, y)=\Sigma_{j} k_{j} t_{j, 1} \cdots t_{j, n}$ we denote $\sum_{j} k_{j}\left(\ldots\left(z, t_{j, 1}\right), \ldots, t_{j, n}\right)$ by $[z \mid f(x, y)]$. Denoting the generators of $E(5,2)$ by 1 and 2 , the additional relations holding in $L(5,2)$ beyond those holding in $E(5,2)$ are

$$
\begin{array}{r|r}
{[((2,1), 1)} & f(1,2)]=0, \\
{[((2,1), 2)} & f(1,2)]=0, \\
{[(((2,1), 1), 2)} & f(1,2)]=0 .
\end{array}
$$

These relations were determined by Havas' program and found to be trivial. It follows that the associated lie ring of $\bar{B}(5,2)$ is $E(5,2)$, whence our result.

\section{Nilpotent group computation methods}

Macdonald [10] and Wamsley [17] have developed algorithms which, given a presentation of a group $G$ and a positive integer $c$, construct the largest nilpotent of class $c$ quotient group of $G$. The treatment here is based upon Wamsley's method.

Starting with a finite presentation of a group

$$
G=\left\langle a_{1}, \ldots, a_{l} \mid R_{1}=R_{2}=\ldots=R_{r}=1\right\rangle,
$$

Wamsley describes a method for computing a consistent commutator power presentation for the largest nilpotent class $c$ quotient group $G / G_{c+l}$.

A commutator power presentation is a presentation of the form

$$
\begin{array}{r}
G=\left\langle a_{1}, \ldots, a_{n}\right| a_{i}^{\rho_{i}}=a_{i+1}^{\alpha(i, i+1)} a_{i+2}^{\alpha(i, i+2)} \ldots a_{n}^{\alpha(i, n)}, 1 \leq i \leq n, \rho_{i}>0 ; \\
\left.\left[a_{j}, a_{i}\right]=a_{j+1}^{\beta(j, i, j+1)} a_{j+2}^{\beta(j, i, j+2)} \ldots a_{n}^{\beta(j, i, n)}, 1 \leq i<j \leq n\right\rangle .
\end{array}
$$

It is not necessary for power relations $\left(a_{i}^{\rho_{i}}=a_{i+1}^{\alpha(i, i+1)} \ldots a_{n}^{\alpha(i, n)}\right)$ to occur for each $i$. 
Given such a presentation for $G$ any element of $G$ can be written in

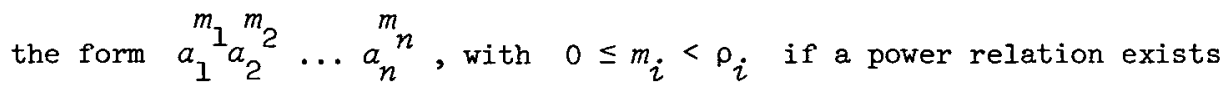
for $i$. This is the normal form. A commutator power presentation is consistent if each element of $G$ can be written uniquely in the normal form.

The method is used in the following way. A consistent commutator power presentation for $G / G^{\prime}$ is computed by abelianizing the relations of the presentation for $G$. If $a_{1}, \ldots, a_{l}$ is the generating set for $G / G^{\prime}$ then $n-l$ relations define $a_{l+1}, \ldots, a_{n}$ in terms of $a_{1}, \ldots, a_{2}$. The other relations are called nondefining.

Suppose a consistent commutator power presentation as above is available for $G / G_{C}$. The process is continued in the following way. New generators $a_{n+1}, a_{n+2}, \cdots$, one for each nondefining relation, are added. For example the sth nondefining relation, say

$$
\left[a_{j}, a_{i}\right]=a_{j+1}^{B(j, i, j+1)} a_{j+2}^{B(j, i, j+2)} \ldots a_{n}^{B(j, i, n)}
$$

would become

$$
\left[a_{j}, a_{i}\right]=a_{j+1}^{\beta(j, i, j+1)} a_{j+2}^{\beta(j, i, j+2)} \ldots a_{n}^{\beta(j, i, n)} a_{n+s} .
$$

This is a commutator power presentation for a maximal class $c l$ generator extension of $G / G_{c}$. It remains to make this presentation consistent, then a presentation for $G / G_{C+1}$.

Wamsley describes a collection process which will convert a word in the $a_{i}$ 's to a word in the normal form in a finite number of steps. He defines an equation to be consistent relative to a presentation if collection of the left hand side yields the same as collection of the right hand side. Then he proves that a commutator power presentation is consistent if and only if the following equations (where brackets indicate order of collection) are consistent:

$$
\left(a_{k} a_{j}\right) a_{i}=a_{k}\left(a_{j} a_{i}\right), I \leq i<j<k \leq n \text {, }
$$




$$
\begin{array}{ll}
\left(a_{k} a_{j}\right) a_{j}^{\rho_{j}-1}=a_{k}\left(a_{j}^{\rho_{j}}\right), & 1 \leq j<k \leq n, \\
\left(a_{k}^{\rho_{k}}\right) a_{j}=a_{k}\left(a_{k}^{\rho_{k}-1} a_{j}\right), & 1 \leq j \leq k \leq n .
\end{array}
$$

Equations (2) and (3) are only required when power relations exist for $a_{j}$ and $a_{k}$, respectively.

The presentation for the extension of $G / G_{C}$ is forced to be consistent by solving these consistency equations. Since the presentation for $G / G_{C}$ was consistent this results in power relations for some of the $a_{n+1}, a_{n+2}, \ldots$. These relations are echelonized. Those with $\rho_{i}$ not equal to 1 are retained in the new presentation while any redundant $a_{i}$ $\left(\rho_{i}\right.$ equal to 1$)$ are eliminated by substitution.

Finally, if $G=\left\langle a_{1}, \ldots, a_{\eta} \mid R_{1}=R_{2}=\ldots=R_{r}=1\right\rangle$, the last step is to ensure that the equations $R_{i}=1$ are consistent relative to the commutator power presentation.

At this stage the process yields a consistent commutator power presentation for $G / G_{C+1}$.

Bayes, Kautsky and Wamsley [3] implemented Wamsley's method for machine computation. They found it convenient to restrict the scope of the program to $p$-groups so that they could extend $G / G_{c}$ by a series of extensions by elementary abelian p-groups, rather than by a full class at a time.

The consistent commutator power presentation for $\bar{B}(5,2)$ given below was computed using essentially that program.

\section{A consistent commutator power presentation for $\bar{B}(5,2)$}

We denote the basic generators of $\bar{B}(5,2)$ by 1 and 2 , and the additional generators introduced by 3 to 34 . For brevity we omit the power relations (which specify that each generator has order 5 ) and those commutator relations that specify that a commutator is trivial. For convenient reference we also list the defining commutator relations 
separately.

The relations which define commutators 3 to 34 are:

$3=[2,1], 4=[2,1,1], 5=[2,1,2], 6=[2,1,1,1]$,

$7=[2,1,2,1], 8=[2,1,2,2], 9=[2,1,2,1,1]$,

$10=[2,1,2,2,1], 11=[2,1,2,1,1,1], 12=[2,1,2,1,1,2]$,

$13=[2,1,2,2,1,1], 14=[2,1,2,2,1,2]$,

$15=[2,1,2,1,1,2,1], 16=[2,1,2,2,1,1,1]$,

$17=[2,1,2,2,1,1,2], 18=[2,1,2,2,1,2,1]$,

$19=[2,1,2,1,1,2,1,1], 20=[2,1,2,2,1,1,2,1]$,

$21=[2,1,2,2,1,2,1,1], 22=[2,1,2,2,1,2,1,2]$,

$23=[2,1,2,1,1,2,1,1,1], 24=[2,1,2,2,1,1,2,1,1]$,

$25=[2,1,2,2,1,2,1,1,1], 26=[2,1,2,2,1,2,1,1,2]$,

$27=[2,1,2,2,1,2,1,2,1], 28=[2,1,2,2,1,2,1,2,2]$,

$29=[2,1,2,2,1,1,2,1,1,1], 30=[2,1,2,2,1,2,1,2,1,1]$,

$31=[2,1,2,2,1,2,1,2,2,1], 32=[2,1,2,2,1,2,1,2,1,1,1]$,

$33=[2,1,2,2,1,2,1,2,2,1,1]$,

$34=[2,1,2,2,1,2,1,2,2,1,1,1]$.

The nontrivial commutator relations are:

$[2,1]=3,[3,1]=4,[3,2]=5,[4,1]=6$,

$[4,2]=7 \cdot 9^{2} \cdot 10 \cdot 11^{4} \cdot 12 \cdot 13^{3} \cdot 14 \cdot 15 \cdot 16^{2} \cdot 17 \cdot 18^{2} \cdot 19^{3} \cdot 21^{2} \cdot 22^{4} \cdot 23 \cdot 24^{3} \cdot 26^{4}$.

$30^{3} \cdot 31^{3} \cdot 32 \cdot 34$

$[4,3]=9^{3} \cdot 11 \cdot 12^{4} \cdot 13^{2} \cdot 15 \cdot 17^{3} \cdot 18^{3} \cdot 19^{4} \cdot 20^{4} \cdot 21^{4} \cdot 22^{4} \cdot 23^{2} \cdot 24^{2} \cdot 25^{2} \cdot 26^{4}$.

$27^{4} \cdot 29^{2} \cdot 30^{2} \cdot 32 \cdot 33^{4}$,

$[5,1]=7,[5,2]=8$,

$[5,3]=10^{4} \cdot 13 \cdot 14^{4} \cdot 15 \cdot 17^{2} \cdot 19 \cdot 20^{2} \cdot 21 \cdot 22^{2} \cdot 23 \cdot 24^{2} \cdot 25^{2} \cdot 26^{3} \cdot 27 \cdot 29 \cdot 30^{2}$.

$31^{2} \cdot 32^{3} \cdot 33$

$[5,4]=12 \cdot 13^{2} \cdot 15^{4} \cdot 16^{3} \cdot 17^{3} \cdot 18^{4} \cdot 19^{4} \cdot 20^{4} \cdot 21 \cdot 23^{3} \cdot 24 \cdot 25^{2} \cdot 26^{4} \cdot 27^{3} \cdot 29^{3}$.

$30^{4} \cdot 32^{4} \cdot 33$

$[6,1]=11^{3} \cdot 16^{3} \cdot 19^{2} \cdot 23^{2} \cdot 24 \cdot 25^{2} \cdot 26^{2} \cdot 29^{2} \cdot 30^{4} \cdot 31 \cdot 32^{3} \cdot 33^{2} \cdot 34^{2}$,

$[6,2]=9^{3} \cdot 11^{3} \cdot 12^{2} \cdot 13^{2} \cdot 15^{2} \cdot 16^{2} \cdot 17 \cdot 18^{2} \cdot 19^{2} \cdot 22 \cdot 23^{2} \cdot 25^{3} \cdot 27^{2} \cdot 29^{3} \cdot 31^{2} \cdot 32^{4}$.

$[6,3]=11^{3} \cdot 15^{4} \cdot 16^{2} \cdot 20^{3} \cdot 21^{3} \cdot 24^{3} \cdot 25^{3} \cdot 27^{4} \cdot 29^{3} \cdot 32^{3} \cdot 33^{3}$,

$33^{3} \cdot 34$,

$[6,4]=19^{2} \cdot 23^{4} \cdot 24^{4} \cdot 25^{4} \cdot 29 \cdot 30^{2} \cdot 32^{2} \cdot 34$,

$[6,5]=15 \cdot 16^{2} \cdot 19^{4} \cdot 21^{4} \cdot 24^{4} \cdot 26^{2} \cdot 27 \cdot 29^{4} \cdot 30^{2} \cdot 31^{3} \cdot 32^{4} \cdot 33 \cdot 34^{3},[7,1]=9$,

$[7,2]=10^{2} \cdot 12 \cdot 13^{2} \cdot 14^{2} \cdot 16 \cdot 17^{2} \cdot 18^{3} \cdot 19 \cdot 20^{4} \cdot 21 \cdot 23^{4} \cdot 24 \cdot 28^{4} \cdot 29^{2} \cdot 30^{3} \cdot 32^{3} \cdot 33^{3} \cdot 34^{4}$, 
$[7,3]=12^{4} \cdot 13^{2} \cdot 15^{4} \cdot 16^{4} \cdot 17^{2} \cdot 19^{3} \cdot 20^{2} \cdot 21 \cdot 25^{3} \cdot 26^{4} \cdot 27^{4} \cdot 29^{2} \cdot 30^{4} \cdot 31^{2} \cdot 32^{3} \cdot 33$,

$[7,4]=15^{2} \cdot 16^{4} \cdot 19^{4} \cdot 20^{3} \cdot 21^{3} \cdot 23^{3} \cdot 25 \cdot 26^{2} \cdot 27 \cdot 29 \cdot 31^{3} \cdot 32^{4} \cdot 33^{3} \cdot 34^{4}$,

$[7,5]=17^{2} \cdot 18 \cdot 20^{2} \cdot 21^{2} \cdot 22^{2} \cdot 24^{3} \cdot 26^{3} \cdot 29 \cdot 30 \cdot 33^{4}$,

$[7,6]=19^{4} \cdot 23 \cdot 24^{3} \cdot 30^{3} \cdot 33 \cdot 34^{3},[8,1]=10$,

$[8,2]=14 \cdot 17^{2} \cdot 18 \cdot 20^{2} \cdot 21^{4} \cdot 22 \cdot 24 \cdot 25^{3} \cdot 26^{4} \cdot 28^{2} \cdot 29^{4} \cdot 30^{3} \cdot 31^{3} \cdot 33^{2} \cdot 34$,

$[8,3]=14^{4} \cdot 17 \cdot 20^{4} \cdot 21^{2} \cdot 22 \cdot 24^{3} \cdot 25 \cdot 26^{3} \cdot 29^{3} \cdot 31 \cdot 32^{2} \cdot 33^{3}$,

$[8,4]=17 \cdot 18^{3} \cdot 20 \cdot 21 \cdot 22^{4} \cdot 24 \cdot 25^{4} \cdot 27 \cdot 30^{3} \cdot 31^{4} \cdot 34^{2}$,

$[8,5]=22^{4} \cdot 26^{3} \cdot 27^{3} \cdot 28^{4} \cdot 30 \cdot 32^{3} \cdot 33^{4} \cdot 34^{2}$,

$[8,6]=20^{4} \cdot 21^{3} \cdot 24^{3} \cdot 25^{3} \cdot 26^{2} \cdot 27^{4} \cdot 29^{3} \cdot 30^{2} \cdot 31^{4} \cdot 32^{2} \cdot 33^{3}$,

$[8,7]=22^{2} \cdot 26 \cdot 27^{3} \cdot 30 \cdot 31 \cdot 32^{2} \cdot 34^{4},[9,1]=11,[9,2]=12$,

$[9,3]=15^{2} \cdot 16^{3} \cdot 19^{2} \cdot 20^{4} \cdot 21^{2} \cdot 23 \cdot 25^{4} \cdot 26^{3} \cdot 27^{4} \cdot 29 \cdot 31^{2} \cdot 32 \cdot 34^{2}$,

$[9,4]=19^{3} \cdot 23^{4} \cdot 25^{3} \cdot 29^{4} \cdot 30^{3} \cdot 32^{3} \cdot 33 \cdot 34$,

$[9,5]=20^{2} \cdot 21 \cdot 24 \cdot 25^{4} \cdot 27^{2} \cdot 30^{4} \cdot 32^{4} \cdot 34,[9,6]=23^{4} \cdot 29 \cdot 32^{2} \cdot 34^{4}$,

$[9,7]=24 \cdot 25^{3} \cdot 29^{3} \cdot 30 \cdot 32^{2} \cdot 34^{3},[9,8]=26 \cdot 27 \cdot 30^{4} \cdot 31^{4} \cdot 32^{3} \cdot 33 \cdot 34^{3}$,

$[10,1]=13,[10,2]=14$,

$[10,3]=17^{4} \cdot 18 \cdot 20^{3} \cdot 21^{4} \cdot 22 \cdot 24^{4} \cdot 25^{3} \cdot 26^{3} \cdot 27^{3} \cdot 29^{3} \cdot 30 \cdot 31 \cdot 33^{4} \cdot 34^{3}$,

$[10,4]=20^{2} \cdot 24^{4} \cdot 26^{3} \cdot 30^{2} \cdot 31 \cdot 34^{2},[10,5]=22^{3} \cdot 26^{3} \cdot 27^{3} \cdot 31^{4} \cdot 34$,

$[10,6]=24^{4} \cdot 25^{3} \cdot 29^{3} \cdot 30^{2} \cdot 32^{4} \cdot 33,[10,7]=26 \cdot 27^{3} \cdot 30^{2} \cdot 31^{4} \cdot 32^{4} \cdot 33 \cdot 34^{2}$,

$[10,8]=28^{4} \cdot 31^{4} \cdot 33 \cdot 34^{3},[10,9]=33^{3} \cdot 34^{2},[11,1]=29 \cdot 32$,

$[11,2]=15^{4} \cdot 16^{2} \cdot 19^{4} \cdot 20^{3} \cdot 21^{3} \cdot 24^{2} \cdot 25^{3} \cdot 26^{4} \cdot 27^{4} \cdot 29 \cdot 30^{4} \cdot 31^{4} \cdot 32^{2} \cdot 33 \cdot 34$,

$[11,3]=19^{4} \cdot 23^{4} \cdot 24^{4} \cdot 25^{4} \cdot 29^{4} \cdot 30 \cdot 32^{2} \cdot 33^{2},[11,4]=23^{4} \cdot 29^{4} \cdot 32 \cdot 34^{2}$,

$[11,5]=24.25^{3} \cdot 29^{2} \cdot 30 \cdot 32 \cdot 34^{2},[11,7]=29.32,[11,8]=30.32^{3} \cdot 33^{4}$,

$[11,10]=32.34^{4},[12,1]=15$,

$[12,2]=17^{2} \cdot 18^{2} \cdot 20^{2} \cdot 21^{2} \cdot 25^{3} \cdot 26^{4} \cdot 28 \cdot 29^{4} \cdot 30^{3} \cdot 32^{3} \cdot 33^{3} \cdot 34^{3}$,

$[12,3]=20^{3} \cdot 26^{3} \cdot 27^{2} \cdot 29 \cdot 30^{2} \cdot 31^{4} \cdot 32^{2} \cdot 33^{2} \cdot 34$,

$[12,4]=24^{4} \cdot 25 \cdot 29 \cdot 30^{4} \cdot 32^{4} \cdot 33^{3},[12,5]=26^{2} \cdot 30^{3} \cdot 31^{4} \cdot 32 \cdot 33^{2} \cdot 34$,

$[12,6]=29 \cdot 32^{4} \cdot 34^{2},[12,7]=30 \cdot 32^{4},[12,8]=31^{4} \cdot 33 \cdot 34^{3}$,

$[12,9]=32^{4} \cdot 34^{4},[12,10]=33^{2},[13,1]=16,[13,2]=17$,

$[13,3]=20^{2} \cdot 21 \cdot 24 \cdot 25^{2} \cdot 26^{2} \cdot 27 \cdot 29^{2} \cdot 31^{4} \cdot 32^{3} \cdot 33^{3} \cdot 34^{3}$,

$[13,4]=24^{3} \cdot 25^{2} \cdot 29^{2} \cdot 30^{3} \cdot 32^{3} \cdot 33 \cdot 34^{4},[13,5]=26^{4} \cdot 30^{3} \cdot 31 \cdot 33 \cdot 34^{3}$,

$[13,6]=29^{4} \cdot 34^{4},[13,7]=30^{3} \cdot 32 \cdot 33^{3} \cdot 34^{3},[13,8]=31^{4} \cdot 33^{2}$,

$[13,9]=32.34^{2},[13,10]=33^{2} \cdot 34,[14,1]=18$,

$[14,2]=22 \cdot 26^{3} \cdot 27^{2} \cdot 28^{4} \cdot 30 \cdot 32^{3} \cdot 33^{4} \cdot 34$,

$[14,3]=22^{4} \cdot 26 \cdot 30^{3} \cdot 31^{3} \cdot 32^{3} \cdot 33 \cdot 34^{3},[14,4]=26 \cdot 27^{3} \cdot 30 \cdot 31 \cdot 33^{4} \cdot 34^{2}$,

$[14,5]=28^{4} \cdot 31^{3} \cdot 33^{2} \cdot 34^{4},[14,6]=30^{3} \cdot 32^{3} \cdot 33^{2} \cdot 34,[14,7]=31^{4} \cdot 33 \cdot 34^{3}$,

$[14,9]=33^{2},[15,1]=19$,

$[15,2]=20^{4} \cdot 21^{2} \cdot 24^{3} \cdot 25^{3} \cdot 26 \cdot 27^{4} \cdot 29^{3} \cdot 30^{2} \cdot 32^{2} \cdot 33^{4} \cdot 34^{3}$, 
$[15,3]=24^{4} \cdot 25^{4} \cdot 29 \cdot 30^{3} \cdot 32 \cdot 33^{2} \cdot 34^{4},[15,4]=29^{3} \cdot 34$, $[15,5]=30^{4} \cdot 32^{2} \cdot 33^{3} \cdot 34^{3},[15,7]=32^{2} \cdot 34,[15,8]=33^{2} \cdot 34^{4}$, $[15,10]=34^{2},[16,1]=34^{2}$, $[16,2]=20^{4} \cdot 21^{4} \cdot 25^{4} \cdot 26^{4} \cdot 27^{3} \cdot 29^{3} \cdot 30 \cdot 31^{4} \cdot 32^{2} \cdot 33^{3} \cdot 34^{4}$, $[16,3]=24^{4} \cdot 25^{4} \cdot 30^{3} \cdot 32^{4} \cdot 33^{2} \cdot 34,[16,4]=29^{4} \cdot 32^{3} \cdot 34^{2}$, $[16,5]=30^{2} \cdot 33 \cdot 34,[16,7]=32^{2} \cdot 34,[16,8]=33^{2} \cdot 34^{4},[16,10]=34^{2}$, $[17,1]=20,[17,2]=22^{4} \cdot 26^{3} \cdot 27 \cdot 28^{2} \cdot 30^{3} \cdot 31^{2} \cdot 32^{3} \cdot 33 \cdot 34^{4}$, $[17,3]=26^{4} \cdot 27 \cdot 30 \cdot 31^{2} \cdot 32^{4} \cdot 33^{2} \cdot 34,[17,4]=30^{2} \cdot 33 \cdot 34^{3}$, $[17,5]=31^{2} \cdot 33^{3} \cdot 34^{3},[17,6]=32^{3} \cdot 34^{2},[17,7]=33^{2} \cdot 34^{4},[17,9]=34^{2}$, $[18,1]=21,[18,2]=22,[18,3]=26^{4} \cdot 27 \cdot 30^{4} \cdot 31^{4} \cdot 32^{3} \cdot 33^{3} \cdot 34^{2}$, $[18,4]=33.34^{2},[18,6]=32^{3} \cdot 34^{2},[18,7]=33^{2} \cdot 34^{4},[18,9]=34^{2}$, $[19,1]=23,[19,2]=25^{3} \cdot 29 \cdot 30^{3} \cdot 32^{2} \cdot 33,[19,3]=29 \cdot 32^{3} \cdot 34$, $[19,5]=32^{2} \cdot 34^{2},[20,1]=24,[20,2]=26 \cdot 27^{3} \cdot 30 \cdot 31 \cdot 32^{2} \cdot 33 \cdot 34$, $[20,3]=30^{4} \cdot 32^{2} \cdot 33^{4},[20,4]=32^{4} \cdot 34^{4},[20,5]=34^{4},[21,1]=25$, $[21,2]=26,[21,3]=30 \cdot 32^{2} \cdot 33 \cdot 34^{2},[21,4]=32^{2} \cdot 34^{4},[21,5]=33^{3} \cdot 34$, $[22,1]=27,[22,2]=28,[22,3]=31.33^{2},[22,4]=33^{3} \cdot 34^{2}$, $[23,2]=29^{4} \cdot 32^{3} \cdot 34^{4},[24,1]=29,[24,2]=30^{4} \cdot 32^{4} \cdot 33$, $[25,2]=30^{4} \cdot 32^{4} \cdot 33^{2} \cdot 34,[25,3]=32^{4} \cdot 34^{2},[25,5]=34^{3}$, $[26,1]=33^{2} \cdot 34,[26,2]=31^{4} \cdot 33 \cdot 34,[26,3]=33^{4} \cdot 34,[26,4]=34^{4}$, $[27,1]=30,[27,2]=34^{3},[27,3]=33^{3} \cdot 34^{2},[27,4]=34^{3}$, $[28,1]=31,[29,2]=32^{4} \cdot 34,[30,1]=32,[30,2]=33^{2} \cdot 34^{3}$, $[31,1]=33,[32,2]=34^{2},[33,1]=34$.

It follows immediately from the consistent commutator power presentation that $\bar{B}(5,2)$ has order $5^{34}$ and class 12 .

\section{A comparison of the methods}

The Lie algebraic computations are easier than the direct group computations. This is to be expected for the lie ring of $\bar{B}(5,2)$ is given by the homogenous components of the consistent commutator power presentation and is thus a sub-part of that presentation. The relative degree of difficulty is illustrated by the timing considerations for the respective computer calculations. The computation of $L(5,2)$ takes 9 CPU seconds while the computation of the consistent commutator power presentation for $\bar{B}(5,2)$ takes 15 minutes on comparable machines.

On the other hand, the consistent commutator power presentation gives the full multiplication table for the group, while the Lie algebra method 
yields merely the Lie ring of the group, and it is very difficult to fully relate the group and its lie ring.

As regards other Burnside groups there is some hope that the Lie algebra methods may yield new information about $\bar{B}(5,3)$ and $\bar{B}(7,2)$.

\section{References}

[1] S.1. Adyan, "Periodic groups of odd exponent", Proc. Second Internat. Conf. Theory of Groups, Canberra, 1973 (Lecture Notes in Mathematics. Springer-Verlag, Berlin, Heidelberg, New York, to appear).

[2] William A. Alford, George Havas and M.F. Newman, "Groups of exponent 4 ", Notices Amer. Math. Soc. 21 (1974), A-291.

[3] A.J. Bayes, J. Kautsky and J.W. Wamsley, "Computation in nilpotent groups (application)", Proc. Second Internat. Conf. Theory of Groups, Canberra, 1973 (Lecture Notes in Mathematics. SpringerVerlag, Berlin, Heidelberg, New York, to appear).

[4] W. Burnside, "On an unsettled question in the theory of discontinuous groups", Quart. J. Pure Appl. Math. 33 (1902), 230-238.

[5] George Havas, "Computational approaches to combinatorial group theory", $\mathrm{PhD}$ thesis, University of Sydney, February, 1974.

[6] A.H. Ностринин [A.l. Kostrikin], "Решение ослабленной проблемы Бернсайда для покаэателя 5 " [Solution of a weakened problem of Burnside for exponent 5 ", Izv. Akad. Nauk SSSR Ser. Mat. 19 (1955), 233-244; MR17,126.

[7] А.Н. Ностринин [A.l. Kostrikin], "О свяэн менцу периодичесними группами и нольцами Ли" [On the connection between periodic groups and Lie rings", Izv. Akad. Nauk SSSR Ser. Mat. 21 (1957), 289-310; Amer. Math. Soc. Trans 2. (2) 45 (1965), 165-189.

[8] А.Н. Костриннн [A.l. Kostrikin], "О проблеме Бернсайда" [The Burnside problem], Izv. Akad. Nauk SSSR Ser. Mat. 23 (1959), 3-34; Amer. Math. Soc. Transl. (2) 36 (1964), 63-99. 
[9] Eugene F. Krause and Kenneth W. Weston, "On the Lie algebra of a Burnside group of exponent 5 ", Proc. Amer. Math. Soc. 27 (1971), 463-470.

[10] I.D. Macdonald, "A computer application to finite p-groups", $J$. Austral. Math. Soc. 17 (1974), 102-112.

[11] П.С. Новинов, С.И. Адян [P.S. Novikov, S.І. Adyan], "О беснонечных пернодичесних группах. I" [Infinite periodic groups. I], Izv. Akad. Nauk SSSR Ser. Mat. 32 (1968), 212-2.44; Math. USSRIzv. 2 (1968), 209-236 (1969).

[12] П.С. Новинов, С.И. АДян [P.S. Novikov, S.I. Adyan], "О беснонечных периоднчесних группах. II" [Infinite periodic groups. II], Izv. Akad. Nauk SSSR Ser. Mat. 32 (1968), 251-524; Math. USSRIzv. 2 (1968), 24I-479 (1969).

[13] П.С. Новинов, С.К. Адян [P.S. Novikov, S.1. Adyan], "О беснонечных периоднчесннх группах. III" [Infinite periodic groups. III], Izv. Akad. Nauk SSSR Ser. Mat. 32 (1968), 709-731; Math. USSRIzv, 2 (1968), 665-685 (1969).

[14] И.H. Санов [I.N. Sanov], "Установление связи между периодичесними группами с пернодом простым чнслом и нольцамн Ли" [Establishment of a connection between periodic groups with period a prime number and Lie rings], Izv. Akad. Nauk SSSR Ser. Mat. 16 (1952), 23-58.

[15] G.E. Wall, "On Hughes' ${ }_{p}$ problem", Proc. Internat. Conf. Theory of Groups, Canberra, 1965, 357-362 (Gordon and Breach, New York, 1967).

[16] G.E. Wall, "On the Lie ring of a group of prime exponent", Proc. Second Internat. Conf. Theory of Groups, Camberra, 1973 (Lecture Notes in Mathematics. Springer-Verlag, Berlin, Heidelberg, New York, to appear). 
[17] J.W. Wamsley, "Computation in nilpotent groups (theory)", Proc. Second Internat. Conf. Theory of Groups, Canberra, 1973 (Lecture Notes in Mathematics. Springer-Verlag, Berlin, Heidelberg, New York, to appear).

School of Information Sciences,

Canberra College of Advanced Education,

Canberra;

Department of Pure Mathematics,

University of Sydney,

Sydney, New South Wales;

School of Mathematical Sciences,

Flinders University of South Australia,

Bedford Park, South Australia. 